\title{
Single Molecule Stepping and Structural Dynamics of Myosin X
}

\author{
Yujie Sun ${ }^{1}$, Osamu Sato ${ }^{2}$, Felix Ruhnow ${ }^{3}$, Mark E. Arsenault ${ }^{1}$, Mitsuo lkebe ${ }^{2}$, and Yale E. \\ Goldman ${ }^{1}$ \\ ${ }^{1}$ Pennsylvania Muscle Institute, University of Pennsylvania, Philadelphia, PA 19104 \\ 2University of Massachusetts Medical School, North Worcester, MA 01655 \\ ${ }^{3}$ Max Planck Institute of Molecular Cell Biology and Genetics, 01307 Dresden, Germany
}

\begin{abstract}
Myosin $\mathrm{X}$ is an unconventional myosin with puzzling motility properties. We studied the motility of dimerized myosin $\mathrm{X}$ using single molecule fluorescence techniques - polTIRF, FIONA, and Parallax to measure rotation angles and 3-dimensional position of the molecule during its walk. It was found that Myosin X steps processively in a hand-over-hand manner following a left-handed helical path along both single actin filaments and bundles. Its step size and velocity are smaller on actin bundles than individual filaments, suggesting myosin $\mathrm{X}$ often steps onto neighboring filaments in a bundle. The data suggest that a previously postulated single $\alpha$-helical domain mechanically extends the 3-IQ motif lever arm and either the neck-tail hinge or the tail is flexible. These structural features, in conjunction with the membrane and microtubule binding domains, enable myosin $\mathrm{X}$ to perform multiple functions on varied actin structures in cells.
\end{abstract}

\section{Introduction}

Myosin X, an unconventional member of the myosin superfamily, is widely expressed in vertebrate tissues. It is found to localize primarily at regions with dynamic actin reorganization, such as the tips of filopodia, the edges of lamellipodia and membrane ruffles ${ }^{1-8}$. At these actinrich protrusions, myosin $\mathrm{X}$ plays a role in filopodia formation, extension and sensing, possibly by transporting actin binding proteins to the tips and by linking membranes and actin ${ }^{2-6,9,10}$. Myosin X is also found to interact with mitotic spindle poles in Xenopus oocytes and plays an important role in maintenance of spindle integrity ${ }^{11-13}$.

The intriguing cellular functions of myosin $\mathrm{X}$ are accomplished by its unique structural features: a motor domain, three calmodulin binding IQ motifs, a putative single $\alpha$-helical domain, a coiled-coil, and a tail containing three PH domains, one MyTH4 domain, and one FERM domain per heavy chain ${ }^{1}$. The PH domains are unique among the myosin super-family and enable myosin $\mathrm{X}$ to directly bind to the plasma membrane ${ }^{2}$ and thereby mediate membranecytoskeleton interactions ${ }^{14}$. The MyTH4-FERM domain enables myosin X to interact with microtubules ${ }^{11}$.

The in vivo and in vitro motility of myosin $\mathrm{X}$ is quite puzzling. Actin based motility of various constructs has suggested that this myosin forms a dimer in the cell and moves processively

Corresponding Author: Yale E. Goldman, Mailing Address: D700 Richards Building, Pennsylvania Muscle Institute, University of Pennsylvania, Philadelphia, PA 19104-6083, Telephone: 215-8984247, Fax: 215-8982653, goldmany@mail.med.upenn.edu.

Author Contributions:

Y.S. carried out the single molecule experiments and data analysis. O.S. prepared and labeled the myosin X protein. F.R. coded Matlab scripts for analysis of FIONA data. M.E.A carried out the bead motility assay on suspended filaments and data analysis. M.I. and Y.E.G supervised the project and, with Y.S., wrote the manuscript. 
toward the barbed ends of actin filaments ${ }^{2,3,15}$. However, Knight et al. have shown that the putative coiled-coil domain of myosin $\mathrm{X}$ is highly charged and unable to dimerize ${ }^{16}$, so myosin $\mathrm{X}$ might require other cellular components to dimerize as has been suggested for myosin $\mathrm{VI}^{17}$. The kinetics of myosin $\mathrm{X}$ are also under debate ${ }^{8,18}$. Nagy et al. showed that myosin $\mathrm{X}$ selects bundled actin for motility and exhibits poor processivity on single actin filaments ${ }^{7}$. However, myosin $\mathrm{X}$ has been found to interact with single actin filaments and possibly move processively to carry cargos or pull cytoskeletal filaments together ${ }^{13,15,18,19}$. Overall, mechanistically relevant information about the motility of myosin X, such as its processivity, step size, and strain-dependent properties, is still largely in question.

In the present work, we used single molecule polarized total internal reflection fluorescence (polTIRF) microscopy $20^{-23}$, fluorescence imaging with one nanometer accuracy (FIONA) ${ }^{24}$, and Parallax (a technique for nanometer tracking of single fluorophores in 3 dimensions $(3 \mathrm{D}))^{25}$ to study the motility of a dimer construct of myosin X on actin filaments and bundles. We observed robust processivity of myosin X on single actin filaments and found that actin bundle formation is not required for processive movement, in contrast to the results of Nagy et al.7. Myosin X walks hand-over-hand along actin filaments with a step size of $34 \mathrm{~nm}$ and demonstrates a left-handed helical path. It moves slower and shows a smaller average step size on actin bundles than on single actin filaments, again following a left-handed helical path. The radii of the helical path on actin filaments and bundles suggest a flexible tail or head-tail junction and the step size measurements support the hypothesis of an $\alpha$-helical extension to the lever arm. These dynamic features and the diversified binding domains in its tail allow myosin $\mathrm{X}$ to interact with multiple binding targets and perform widely varied functions in crowded, actin-rich regions of the cell.

\section{Results}

\section{Myosin X moves processively on both actin filaments and bundles}

A recombinant bovine myosin X HMM construct, dimerized by the myosin $\mathrm{V}$ coiled-coil (Myo10-HMM-M5cc)15, 18, 19, was used in most of the experiments. Myo10-HMM-M5cc was labeled either with bifunctional rhodamine (BR) on one of the calmodulin $(\mathrm{CaM})$ subunits (termed MyoX-BRCaM), with a quantum dot (QD655, Invitrogen) on a CaM in the lever arm (termed MyoX-LA-QD655), or at the C-terminus of the heavy chain (termed MyoX-CT-

QD655). Single molecule motility assays showed that myosin X moves processively on both single actin filaments and fascin-actin bundles, with characteristic run lengths of about $1 \mu \mathrm{m}$ both at low $(0.5 \mu \mathrm{M})$ and saturating $(2 \mathrm{mM}) \mathrm{MgATP}$ concentrations and when actin was either bound to the surface or suspended above a $2 \mu \mathrm{m}$ gap across microfabricated polymer ridges (Figs. 1a-b, Supplementary Movies 1 and 2 and Table 1). Therefore, immobilization of actin filaments or bundles onto the microscope slide does not appreciably decrease the processivity of myosin X. The stepping rate of myosin X depends on ATP concentration (Fig. 1c), leading to rate constants for ATP binding ( $\left.\mathrm{k}_{\mathrm{ATP}+}\right)$ of $1.24 \mu \mathrm{M}^{-1} \mathrm{~s}^{-1}$ and an ATP independent step (presumably ADP release, $\mathrm{k}_{\mathrm{ADP}-}$ ) of $6.90 \mathrm{~s}^{-1}$. These values were $1 / 2-1 / 3$ of those previously obtained values for the single headed construct ${ }^{18}$, likely due to temperature differences in the experiments and inter-head tension (gating) in the dimer molecule, as previously shown for myosin $\mathrm{VI}^{26}$.

The construct used for the experiments above was forced to dimerize by inclusion of the myosin $\mathrm{V}$ coiled coil tail $(\mathrm{M} 5 \mathrm{cc})$ in its sequence. In order to test whether the artificial dimerization altered the motility relative to a more native construct, we also investigated the motility of GFP-myosin X HMM which does not spontaneously dimerize in vitro ${ }^{2}, 3,15$. Following experiments on myosin VI17, we tested whether bringing monomeric GFP-myosin X HMM molecules close to each other by binding to actin filaments in the absence of ATP would initiate their dimerization. Native myosin X monomers did dimerize by proximity and, when ATP was 
subsequently added, moved processively along actin (Supplementary Fig.1, Movie 3). Most of the remaining data were obtained from a construct containing the M5cc tail, which facilitates dimerization, but has motility similar to the native proximity-induced dimer.

\section{Myosin X walks hand-over-hand along actin filaments with a $\mathbf{3 4} \mathbf{n m}$ step size}

In a polTIRF experiment, the BR in a MyoX-BRCaM molecule is excited by an evanescent field that has time-multiplexed polarizations. The polarized fluorescence intensities in a cycle of excitation polarizations are used to compute the orientation of the BR, defined by the axial angle $\beta$ (probe polar angle relative to the actin axis) and $\alpha$ (azimuthal probe angle around actin) $20,22,30$. Single molecule polTIRF recordings of MyoX-BRCaM at $2 \mu \mathrm{M}$ MgATP show the fluorescence intensities detected by the $x$ and $y$ polarized photon counters for each excitation polarization (Supplementary Fig. 2a). Abrupt changes of photon counts per detection interval in the individual polarized fluorescence intensity traces, without substantial change of the total photon counting rate, indicate that the labeled lever arm tilts during the processive motility, as shown by the corresponding $\beta$ and $\alpha$ traces (Fig. 2a).

The distribution of $\beta$ angles of all moving myosin $X$ molecules $(N=180)$ has two peaks at $42^{\circ}$ and $135^{\circ}$ (Fig. 2b), suggesting that the stepping of myosin $X$ involves two states, similar to the polTIRF results of myosin $\mathrm{V} 23$ and myosin $\mathrm{VI}^{20}$. If the probe dipole is out of the plane of the lever arm tilting, the probe azimuth $(\alpha)$ will tilt during a step, even if the molecule walks straight along actin. About $90 \%$ of the step transitions of $\beta$ and $\alpha$ occurred simultaneously with approximately equal numbers showing correlated (increase (decrease) of $\beta$ with increase (decrease) of $\alpha, 2^{\text {nd }}$ half of the traces in Fig. 2a) or anti-correlated ( $1^{\text {st }}$ half of the traces in Fig. 2a). The numbers of correlated and anti-correlated transitions are nearly equal probably due to ambiguity from the 2-fold symmetry of the probe dipole of $\mathrm{BR}^{20,30}$.

If a myosin head alternates between leading and trailing positions, then the lever arm returns to its original state after two steps. In that circumstance, $\alpha$ will return to its former value after two steps if the molecule is walking straight, regardless of whether the probe is oriented in the tilting plane or not. The change of $\alpha$ after two steps, termed ${ }^{2} \Delta \alpha$, can thus be used to evaluate whether the motor walks straight along the axis of $\operatorname{actin}^{20}$. The ${ }^{2} \Delta \alpha$ distribution of myosin $\mathrm{X}$ (Fig. 2c), centered near zero, is approximately as narrow as that of myosin V (Supplementary Fig. 2b) and about half as wide as that of myosin $\mathrm{VI}^{20}$. These results indicate that myosin $\mathrm{X}$, like myosin $\mathrm{V}$, steps mostly straight, unlike the chaotic side-to-side motions of myosin $\mathrm{VI}^{20}$.

High precision single molecule tracking of fluorophores (FIONA24) on the lever arms of myosin V24 and VI32,33 has shown that these isoforms step in a hand-over-hand manner based on the observation of alternating larger and smaller displacements of the fluorophore. We find a similar stepping pattern of MyoX-LA-QD655 (Fig. 3). 86\% of myosin X molecules showed clear alternating $\sim 25 \mathrm{~nm}$ and $\sim 45 \mathrm{~nm}$ displacements (Fig. 3a, $1^{\text {st }}$ and $2^{\text {nd }}$ Panels). The rest of the MyoX-LA-QD655 molecules demonstrated larger step sizes with a narrower distribution at $68 \pm 8.4 \mathrm{~nm}$, (s.d., Fig. 3b).

The step size distribution of the alternating molecules ( $3^{\text {rd }}$ panel of Fig. 3a) could be fitted by a single Gaussian curve at $34 \pm 14 \mathrm{~nm}$ (s.d.). However, due to the clear alternation of step sizes in these molecules (typical recording in the $1^{\text {st }}$ and $2^{\text {nd }}$ panels in Fig. 3a), producing two populations of steps in equal numbers, we fit the distribution with two Gaussian components having equal area (amplitudes and standard deviations of the two components were forced to be equal). The fitting yielded peaks centered at $25 \mathrm{~nm}$ and $43 \mathrm{~nm}$, which in conjunction with the relatively large size of the quantum $\operatorname{dot}(\sim 15 \mathrm{~nm})$, suggest that the quantum dot is probably located on the second and/or third calmodulin in these molecules (cartoon panel of Fig. 3a). The average of the two alternating step sizes suggests that myosin X's center of mass translocates about $34 \mathrm{~nm}$ on each step. This interpretation is confirmed by the step size 
measurements of MyoX-CT-QD655 (Fig. 4), which indicates the motion of the tail of the molecule, tracking the center of mass.

The myosin $\mathrm{X}$ molecules producing $68 \mathrm{~nm}$ steps probably have a quantum dot located on the first $\mathrm{CaM}$ and thus alternated between 0 and $68 \mathrm{~nm}$ steps (cartoon panel of Fig. 3b). Since 0 $\mathrm{nm}$ steps are not discernible, each observed $68 \mathrm{~nm}$ step is limited by two consecutive events, the steps of the labeled and unlabeled heads. In fact, the dwell time distributions of the two types of myosin X (bottom of Fig. 3) strongly support that interpretation. The dwell time distribution for the $25-43 \mathrm{~nm}$ molecules (red bars in Fig. 3a, bottom) can be fitted by a single exponential $P(t)=k e^{-k t}$, consistent with the labeled and unlabeled heads having the same stepping rate. The dwell time distribution for the $0-68 \mathrm{~nm}$ molecules (blue bars in Fig. 3b, bottom) is fitted better by the convolution of two exponentials $P(t)=t k^{2} \mathrm{e}^{-k t}$, as expected from two reactions with equal rates in series ${ }^{24}$. These results strongly support the hand-over-hand model of myosin X processivity.

Processive motility shows quantitative differences on fascin-actin bundles compared to single actin filaments. Myosin X moves about $26 \%$ faster on actin filaments than on fascin-actin bundles at low and saturating ATP concentrations $([\mathrm{MgATP}]=0.5 \mu \mathrm{M}-2 \mathrm{mM}$, Supplementary Table 1). On bundled actin, the center of mass of myosin X (tracked on MyoX-CT-QD655) showed a smaller average step size ( $27 \mathrm{~nm}$, Fig. $4 d$ ) than that on single filaments ( $34 \mathrm{~nm}$, Fig. $4 c$ ), which can partly be attributed to more backward steps ( $8.0 \%$ of all steps on bundled actin vs. $4.5 \%$ on single filaments) and partly to a higher proportion of short steps $(\sim 20 \mathrm{~nm})$ on bundled actin (Fig. 4d). The ratio of the average step sizes (including short and backward steps) on single filaments and bundled actin is 1.26 , consistent with the difference in myosin $\mathrm{X}$ velocities. The average size of myosin X's backward steps is also different on bundled actin $(17 \mathrm{~nm}$, Fig. 4d) and single filaments (29 nm, Fig. 4c).

In order to test whether these differences in velocity and back-stepping are caused by polarity variations of the actin within bundles, we characterized the fascin-bundled actin using transmission electron microscopy (Materials and Methods and Supplementary Fig. 3). The number of filaments per bundle was found to be $8.5 \pm 0.4 \mathrm{~nm}$ and the actin center-to-center spacing in bundle was $10.6 \pm 0.1 \mathrm{~nm}$ (s.e.m, $n=39$ bundles). Decoration of the actin filaments in bundles with myosin II MgSubfragment 1 (Supplementary Fig. 3c and d) showed that all of the filaments in the bundles are polarized in the same direction ( $n=18$ bundles), consistent with a previous report ${ }^{34}$. Therefore, the backward steps observed were not the result of myosin $\mathrm{X}$ switching onto filaments with opposite polarity. Rather, the stepping dynamics suggest that myosin $\mathrm{X}$ takes long, straight steps along individual actin filaments in bundles, but sometimes steps sideways onto a neighboring filament in the bundle, producing a smaller step, either forward or backward.

We also measured the velocity of native myosin $\mathrm{V}$ on actin bundles and single actin filaments at $1 \mu \mathrm{M}$ and $500 \mu \mathrm{M} \mathrm{MgATP}$. At both ATP concentrations, myosin $\mathrm{V}$ moves about $36 \%$ faster on single actin filaments than on bundles, which is due to more short steps on bundles (Supplementary Fig. 4). Nevertheless, myosin V produces fewer backward steps than myosin $\mathrm{X}$ on both types of track.

\section{Myosin X walks in a left-handed helical path along actin}

The $34 \mathrm{~nm}$ step size suggests that the trailing and leading heads of myosin $\mathrm{X}$ bind actins separated by $11(31 \mathrm{~nm})$ and $13(36 \mathrm{~nm})$ actin monomers. For these spans, the myosin molecules would not be subjected to high intra-molecular strain due to large azimuthal differences between other pairs of actin binding sites ${ }^{20,35}, 36$. Stepping on the $11^{\text {th }}$ and $13^{\text {th }}$ actin monomers, myosin $\mathrm{X}$ should exhibit a net left-handed twirling path along actin ${ }^{37}$. In conventional in vitro processive motility assays, though, actin is usually fixed onto a substratum 
so that the myosin molecules cannot access the space underneath the actin, preventing full rotations of helical motion.

We suspended actin filaments and bundles over microfabricated trenches (Supplementary Fig. 5 ) and used the Parallax technique ${ }^{25}$ to study the $3 \mathrm{D}$ motion of myosin $\mathrm{X}$. The microstructures were rows of polymethyl methacrylate (PMMA) ridges and grooves at $3 \mu \mathrm{m}$ pitch. The grooves were measured to be $2 \mu \mathrm{m}$ wide and $350 \mathrm{~nm}$ deep by AFM contact mode imaging (Supplementary Fig. 5b). Actin was suspended over the grooves and pulled taut by hydrodynamic flow (Materials and Methods). Images with TIRF and Low Angle Oblique illumination ${ }^{38}$ confirmed that most of the filaments were suspended away from the slide surface (Supplementary Fig. 5)

MyoX-LA-QD655 (Fig. 5a) and MyoX-CT-QD655 (Fig. 5b and c) were observed stepping along suspended actin filaments and fascin-actin bundles (Supplementary Movie 2). In each case in Fig. 5, the upper panel shows the displacement of myosin $\mathrm{X}$ along the actin aligned with the $x$ direction in the microscope, the middle panel is the lateral displacement of the quantum dot relative to the actin in the $y$ (blue) and $z$ (red) directions. The lateral displacements are fitted with sinusoidal functions to obtain their periods, amplitudes, and phases. The lower panels are 3D plots of the $x, y$ and $z$ displacements. The green line represents the actin position, the black traces are the trajectories of myosin X relative to actin, and the red trace represents the sinusoidal fitted path. In all three examples, the $z$ displacement leads the $y$ displacement by approximately a quarter of a period, which indicates that the paths are left-handed helices.

Of 465 MyoX-LA-QD655 and MyoX-CT-QD655 molecules moving on suspended actin filaments, $308(66 \%)$ followed helical paths around actin, the rest traveling approximately straight over their run lengths. In contrast, on actin bundles, only 28\% myoX-CT-QD655 molecules demonstrated helical motion; most of the molecules walked straight. The mean helical pitch in the three cases are all close to $1 \mu \mathrm{m}$ (MyoX-LA-QD655 on actin filaments: $1018 \pm 37 \mathrm{~nm}$ (s.e.m, $n=331$ ), MyoX-CT-QD655 on actin filaments: $1042 \pm 67 \mathrm{~nm}(n=134)$, and MyoX-CT-QD655 on actin bundles: $1037 \pm 49 \mathrm{~nm}(n=40))$, which is slightly smaller than the value, $1296 \mathrm{~nm}$, expected from the step size of $34 \mathrm{~nm}^{39}$. This difference is probably due to the average run length of myosin X (Fig. 1 and Supplementary Table 1) being similar to its helical pitch causing many short runs with long helical pitches not to show periodic deflections thus excluding them from the pitch analysis. To quantify the pitch, we included only molecules whose run length was one half of the helical turn or more. Myosin X may also have a longer true helical pitch on bundled actin than on single filaments so that many molecules dissociated from actin before they finished moving half of their helical path, and thus were categorized into the group of straight-walkers. This effect would result in fewer helically walking molecules on bundles (28\%) than on single filaments (66\%).

The mean radii of the helical paths are significantly different for the two labeling positions and between actin filaments and bundles (Fig. 6). The path of the probe of MyoX-LA-QD655 on actin filaments had radius $20.1 \pm 1.0 \mathrm{~nm}$ (s.e.m, $n=331$ ), approximately the value expected, as illustrated in the leftmost cartoon of Figure 6. The mysoin V coiled-coil (183 residues) in between the head-tail junction and the C-terminus of myoX-CT-QD655 would extend the radius $\sim 27 \mathrm{~nm}$ if it were straight (middle cartoon in Fig. 6). However, the radius of the probe path of MyoX-CT-QD655 on actin filaments was $27.1 \pm 3.1 \mathrm{~nm}$ (s.e.m, $n=134$ ) only $7 \mathrm{~nm}$ greater than that of myoX-LA-QD655, suggesting that the coiled-coil is not straight or oriented radially, presumably due to flexibility at the head-tail junction or farther along the sequence. The helical radius of MyoX-CT-QD655 on bundles of approximately 9 actin filaments was $37.4 \pm 4.7 \mathrm{~nm}$ (s.e.m, $n=40$ ), $10 \mathrm{~nm}$ larger than that on individual filaments, reasonably consistent with the increased radius of the bundles (rightmost cartoon in Fig. 6). We also measured the path of polystyrene beads ( $1 \mu \mathrm{m}$ diameter, Polysciences, Inc.), heavily coated 
with myosin $\mathrm{X}$, along actin filaments which were suspended between gold electrodes using dielectrophoresis in an AC electric field ${ }^{40}, 41$. Myosin X coated beads followed left-handed helical paths with a pitch $1.5 \pm 0.4 \mu \mathrm{m}$ (s.d., $n=4$ ) (Fig. $5 \mathrm{~d}$ ).

\section{Discussion}

Our results show that myosin $\mathrm{X}$ moves processively on individual actin filaments, with an average step size of $34 \mathrm{~nm}$, and more slowly on actin bundles with an average step size of 27 $\mathrm{nm}$. A processive myosin motor requires a high duty ratio and a sufficiently long lever arm to stride between appropriately oriented binding sites on the helical actin track. The myosin X construct used in this study was determined to have a high duty ratio ${ }^{18}$ although a bovine myosin X S1 construct produced a low duty ratio ${ }^{8}$. This discrepancy has been discussed by Homma and Ikebe ${ }^{18}$. The robust processivity reported here implies a high duty ratio in our construct.

Each myosin $\mathrm{X}$ lever arm has three IQ motifs (extending about $10.8 \mathrm{~nm}$ ), so a myosin $\mathrm{X}$ dimer would be expected to stride maximally over $22 \mathrm{~nm}$ if there were no further extension to its lever arm or unraveling of its proximal tail. Knight et al. ${ }^{16}$ have suggested that the proximal tail domains of myosin X form non-dimerizing stable single $\alpha$-helices (SAH domains) that act as mechanically stiff extensions to the myosin $\mathrm{X}$ lever arm. The SAH sequence (55 residues following the 3rd IQ motif) and the distal coiled-coil domain (the next 78 residues) are both present in the construct mainly used here, followed by the coiled-coil domain from myosin $\mathrm{V}$ to force dimerization.

The $34 \mathrm{~nm}$ step size of myosin $\mathrm{X}$ on actin filaments (Figs. 3 and 4) supports the assumption that there are extensions to the lever arm of myosin $\mathrm{X}$ beyond the $10.8 \mathrm{~nm}$ length of its three $\mathrm{CaM}$ binding sites. The postulated 55-residue SAH domain may increase the length of lever arm by approximately $7.5 \mathrm{~nm}^{16}$ and allow the two heads of myosin $\mathrm{X}$ to bind 11 or 13 actin monomers apart, a sterically favorable configuration.

Myosin VI is another myosin motor that is thought to have extensions to its lever arms and thereby reach a large step size ${ }^{20,26,42}$. However, evidence from different measurements, including angles and step sizes, suggests there are differences in the rigidity of the lever arm of myosin V, VI, and X. ${ }^{2} \Delta \alpha$, the change of the probe azimuthal angle $\alpha$ after two steps, is related to the path of a moving myosin motor ${ }^{20}$. The ${ }^{2} \Delta \alpha$ distribution of myosin VI was found to be much wider $\left(\sigma=55^{\circ}\right)^{20}$ than that of myosin V $\left(\sigma=29^{\circ}\right.$, Supplementary Fig. $\left.2 b\right)$ or X ( $\sigma$ $=30^{\circ}$, Fig. 2c), suggesting that myosin VI has more flexible lever arms and walks more wiggly on actin filaments ${ }^{20}$. The step size distributions of the three myosin motors are consistent with these angular measurements. Stepping distance of fluorescent labeled myosin $\mathrm{X}$ heads (twice the center of mass motion, $68 \pm 8.4 \mathrm{~nm}$, (s.d., $n=1168$, Fig. 3b)) has variability closer to that of myosin V (74 \pm 5.3 nm, s.d. ${ }^{24}$, and $74 \pm 7.7$ nm, s.d., $n=140$, Supplementary Fig. 4 a), than to myosin VI ( $70 \pm 23 \mathrm{~nm}$,s.d.32). The highest variability in the step size of myosin VI is likely due to its high flexibility. Lever arm flexibility may also affect the likelihood of backward steps. Myosin VI exhibits more backward steps33,43 than myosin V (Ref.24 and Supplementary Fig. 4). In our step size measurements on myosin X (Fig. 4) and V (Supplementary Fig. 4), we found that myosin X also has more backward steps than myosin $\mathrm{V}$.

The differences in the lever arm rigidity of the three myosins are a consequence of their structures. Myosin $\mathrm{V}$ has rigid lever arms with six bound $\mathrm{CaMs}$ and produces robust processive stepping. In order to achieve their large step sizes, however, both myosin VI and X need extensions to their short lever arms which seem to increase the overall flexibility. Part of the extended myosin VI lever arm may contain a random coil ${ }^{20,26,33}$ or an unfolded three-helix 
bundle ${ }^{42}$, which confers high flexibility. In contrast, myosin X only has one SAH to extend its three-CaM bound lever arm. The $\mathrm{SAH}$ is expected to be less rigid than the lever arm of myosin $\mathrm{V}$, but more rigid than the extension of myosin VI. Thus the SAH domain of myosin X is likely to carry mechanical load and facilitate intra-molecular gating ${ }^{44}$.

Myosin X moves more slowly on fascin-actin bundles than it does on actin filaments, consistent with its smaller average step size of $27 \mathrm{~nm}$ on bundled actins (Fig. $4 \mathrm{c}$ and d). The variable step sizes and left-handed helical walking path of myosin X on actin bundles (Figs. 5 and 6) suggest that myosin X switches onto adjacent actin filaments rather randomly. This contrasts with the more predictable "straddle mechanism" proposed by Nagy et $\mathrm{al}^{7}$, in which the two heads of a myosin X necessarily track along two adjacent actin filaments. Our measurements show that myosin $\mathrm{V}$ also moves more slowly on actin bundles than on single filaments. The multiple actin binding sites available in a bundle seem to deflect myosin $\mathrm{V}$ and $\mathrm{X}$ from a straight path, producing slower velocities. Nevertheless, it may be advantageous that myosin $\mathrm{X}$ is able to switch onto adjacent actin filaments in a bundle or span between two actin filaments. For instance, this feature may allow myosin X to bypass "road blocks" on actin bundles during cargo transport in a crowded filopodia. Myosin X is able to pull actin filaments together at the cell periphery to initiate filopodial actin bundles ${ }^{15}$, implying that myosin X can span across actin filaments.

Processive motility after dimerization of the native tail sequence by clustering on actin shows that processive motility of myosin $\mathrm{X}$ on individual actin filaments is a feature of the native molecule when two motor domains are paired. The robust processivity we observed on individual actin filaments contrasts with the findings of Nagy et al.7, who reported that myosin $\mathrm{X}$ preferentially selects bundled actins for motility and shows poor processivity on single filaments. These differences are presumably due to the different truncation points in the coiledcoil domain or dimerization sequences and positions used in the two studies. The myosin $\mathrm{X}$ construct of Nagy et al. ${ }^{7}$ used a GCN4 leucine zipper to ensure dimerization of the heavy chains rather than the myosin $\mathrm{V}$ tail used here. The GCN4 was placed in almost the same position (4 residues later) as the start of our myosin $\mathrm{V}$ tail. The stability and flexibility of the putative $\mathrm{SAH}$ domain may depend on details or the position of the following coiled coil and an unstable domain structure might diminish gating within myosin X. Alternatively, it is plausible that a leucine zipper might decrease the flexibility of the neck for finding appropriate binding sites on actin filaments, thus reducing the processivity. The radii of helical paths for CaM labeled and C-terminally labeled myosin X (Figs. 5 and 6) imply that the tail of our construct is flexible.

Myosin X has been found to walk on both individual actin filaments ${ }^{13,15,18,19}$ and actin bundles $^{7}$ in cells as we found in vitro. The postulated cellular functions of myosin $\mathrm{X}$ are surprisingly varied, probably requiring bivalent association with different actin filaments in formation of filopodia ${ }^{15}$, membrane interactions in the transport of integrins ${ }^{4}$ and growth factor receptors $^{3}$ to filopodial tips and in amplifying morphogenic signals ${ }^{9}$, and possibly microtubule binding in maintenance of nuclear position and the mitotic or meiotic spindle ${ }^{11-13}$. The flexible tail or head-tail junction in myosin $\mathrm{X}$ may enable interactions between its head and tail domains to regulate its activity, like myosin $\mathrm{V}^{45,46}$ and would also allow myosin $\mathrm{X}$ to adopt different geometries in complex, more or less dense, actin structures in the lamellipodia, filopodia, near the nucleus, and the spindle. The semi-flexible nature of the SAH domains and tail may allow myosin $\mathrm{X}$ to stretch, and pull multiple actin filaments into bundles at the base of filopodia ${ }^{15}$ and to squeeze through narrow cytoplasmic spaces during transport in the cell body or filopodia. It is conceivable that a myosin X carrying integrins or other cargos into a filopodium would adopt a spiral motion to ensure location of a substrate for attachment.

Conversion of non-processive monomers into processive dimers has been observed for myosin VI and postulated to be a regulatory feature of other myosins ${ }^{17,27-29}$, Our observation that 
native myosin $\mathrm{X}$ monomers are not processive, but undergo proximity-induced dimerization and thereby become processive provides support for that postulate. The monomer-dimer conversion may regulate the local cellular function of myosin $\mathrm{X}$ as a structural anchor or a cargo transporter.

\section{Conclusions}

In this study, we used single molecule fluorescence techniques, polTIRF, FIONA, and Parallax to investigate the motility of myosin X on actin filaments and bundles. Myosin X moved processively on both single actin filaments and bundles in a hand-over-hand manner. On actin bundles, myosin $\mathrm{X}$ often switched tracks onto adjacent actin filaments and demonstrated slower velocity than on single filaments. The trajectories of myosin X moving freely around suspended actins followed left-handed helical paths, which is consistent with the $34 \mathrm{~nm}$ step size. Our data support the hypothesis of single $\alpha$-helical extensions to the lever arms and suggest a flexible head-tail junction. These structural and dynamical features provide myosin $\mathrm{X}$ adaptability in movement and binding configuration on different structures of the actin cytoskeleton and for its highly varied cellular functions.

\section{Materials and Methods}

\section{Preparation of Proteins}

Expression, purification and labeling of myosin $\mathrm{X}$-We expressed and purified native myosin X (including AA 1 - 979) and recombinant myosin X constructs, Myo10-HMM-M5cc (for MyoX-LA-QD655) and Myo10-HMM-M5cc-BAP (for MyoX-CT-QD655), in SF9 cells as described previously $15,18,19$. The native myosin $X$ has His and FLAG tags at the Nterminus, followed by a GFP motif (GFP-Myosin X HMM). We performed biotinylation of Myo10-HMM-M5cc-BAP on the FLAG resin using $0.01 \mathrm{mg} \mathrm{ml}^{-1}$ biotin protein ligase (Avidity, LLC) in a solution containing $50 \mathrm{mM}$ Bicine (pH 8.3), $10 \mathrm{mM}$ ATP, $10 \mathrm{mM} \mathrm{Mg}$ acetate, $50 \mu \mathrm{M}$ biotin and $1 \mathrm{mM}$ DTT on ice overnight. For polTIRF experiments, we labeled myosin X with bifunctional rhodamine (BR-I2, a generous gift from Dr. J.E.T Corrie ${ }^{47}$, MRC National Institute for Medical Research, London, UK) as previously for myosin V23 and VI20.

In separate experiments, we labeled myosin $\mathrm{X}$ with streptavidin coated quantum dots QD655 (Invitrogen, Inc.) via biotin-streptavidin linkage to a calmodulin $(\mathrm{CaM})$ or the $\mathrm{C}$-terminus of the heavy chain. We prepared biotinylated CaM (biotin-CaM) with P66C chicken CaM mutation $^{23}$ and biotin-maleimide (Sigma) essentially as described by Dunn and Spudich48.

Actin filaments and fascin-actin bundles-We purified G-actin from rabbit skeletal muscle as described by Pardee and Spudich ${ }^{49}$. We prepared Alexa647-labeled F-actin (Gactin:Alexa647 = 5:1) and rhodamine-phalloidin labeled F-actin as described previously 20 , 30. We expressed and purified fascin in SF9 cells50. We prepared actin bundles by mixing and incubating $3 \mu \mathrm{M}$ fascin and $8 \mu \mathrm{M}$ F-actin on ice for 2 days7.

\section{Experimental Apparatus and Assays}

Single molecule poITIRF assay-We prepared flow chambers by assembling a fused silica slide (Quartz Scientific) with a glass coverslip using double-sided adhesive tape. We immobilized actin on the surface by successive incubations with NEM-Myosin II (60 nM, 5 $\mathrm{min}$ ) and Alexa647-labeled F-actin (100 $\mathrm{nM}, 1 \mathrm{~min})$. We blocked the surface using $5 \mathrm{mg}$ $\mathrm{ml}^{-1}$ casein (5 min). Finally, we introduced $100 \mathrm{pM}$ BR-CaM labeled myo10-HMM-M5cc in the motility buffer (M5Buffer: $25 \mathrm{mM} \mathrm{KCl}, 20 \mathrm{mM}$ Hepes $\mathrm{pH}$ 7.6, $5 \mathrm{mM} \mathrm{MgCl}_{2}, 1 \mathrm{mM}$ EGTA, $10 \mathrm{mM}$ dithiothreitol (DTT), $100 \mu \mathrm{g} \mathrm{ml}^{-1}$ wild-type CaM, $10 \mathrm{mM}$ phosphocreatine (Sigma 
P-7936), $0.3 \mathrm{mg} \mathrm{ml}^{-1}$ creatine phosphokinase (prepared daily from powder, Sigma C3755), and the desired amount of ATP).

In previous studies ${ }^{20,22,30}$, we described our single molecule polTIRF setup, which allows unambiguous resolution of individual probe dipole orientations within a hemisphere. Temporal resolution here was $80 \mathrm{~ms}$.

Single molecule in vitro motility assay-We used an inverted Nikon TE-2000U microscope with objective-type (Nikon 100×, 1.49 NA objective) total internal reflection fluorescence (TIRF) excitation from a solid state 488-nm laser (Coherent, Inc.), a solid state 532-nm laser (CrystaLaser, Inc.) and a back-illuminated electron multiplier-CCD camera (Cascade-512B, Photometrics, Inc.).

The flow chamber assembly and immobilization of actin (filaments and bundles) were similar to that in the single molecule polTIRF assay except the fused silica slide was replaced by a glass slide. The motility mixture contained $\sim 600 \mathrm{pM}$ of QD655 labeled myosin X in M5Buffer and an oxygen scavenging system (glucose oxidase, catalase and glucose 51).

Actin-assisted dimerization of Myosin X HMM-To enable proximity-induced dimerization of native myosin $\mathrm{X}$, we first immobilized $800 \mathrm{nM}$ actin filaments on a flow chamber surface, pre-coated with NEM-myosin $(30 \mathrm{nM})$ for $3 \mathrm{~min}$. Then we added $200 \mathrm{nM}$ myosin X HMM and incubated for $5 \mathrm{~min}$ in the absence of ATP. Lastly, we flowed in M5Buffer containing $10 \mu \mathrm{M}$ ATP to wash out the unbound myosin $\mathrm{X}$ and initiate motility of myosin $\mathrm{X}$ dimers. We conducted control experiments by flowing $5 \mathrm{nM}$ myosin X HMM into the flow chamber in the presence of $10 \mu \mathrm{M}$ ATP. We quantified the number of myosin X molecules in moving fluorescent spots by comparing their GFP fluorescence intensity to that of monomeric myosin X, containing one GFP molecule, stuck on the surface.

Characterization of fascin-actin bundles-We used a transmission electron microscope (JEM 1011, JEOL USA) operated at $100 \mathrm{kV}$ to characterize fascin-actin bundles, which were adhered to carbon coated grids and negatively stained with uranyl acetate. For most of the actin bundles, we could identify individual filaments in the bundle (Supplementary Fig. 3a). Crosslinking fascin molecules were sometimes recognized as periodic densities

(Supplementary Fig. 3b). We also investigated whether actin filaments are uniformly polarized within bundles by decorating with myosin II MgSubfragment 1 (Supplementary Fig. 3c and d).

3D tracking on suspended actin filaments and bundles-We developed a 3D tracking technique, Parallax, based on a stereoscopic split-view of the sample, to track the motion of QD655 labeled myosin X molecules along suspended actin. The details of the Parallax setup and calibrations are described in ${ }^{25}$.

Myosin $\mathrm{X}$ is constrained from exploring all the way around surface bound actin filaments. In the 3D tracking experiments, we suspended actin filaments or bundles across microfabricated polymethyl methacrylate ridges so that there was enough clearance between the substrate and suspended actin, see Supplementary Fig. 5. We prepared the microstructures using a micromolding technique ${ }^{52,53}$, described with Supplementary Fig. 5.

In order to suspend actin filaments taut over the microfabricated structures, we added three successive $20 \mu \mathrm{l}$ aliquots of actin $(500 \mathrm{nM})$ into the chamber at intervals of 15 seconds. The high concentration and short incubation time caused many of the actin filaments to bind to the NEM-myosin coated ridges as they were drawn across the pedestals by the flow. For both 2D and 3D single molecule position data, we tracked the QD655 labeled myosin X using an in- 
house written ImageJ plugin or a Matlab tracking program ${ }^{54}$. For QD655 in Parallax tracking, the uncertainty (s.d.) of location was about $4 \mathrm{~nm}$ in the $x$ and $y$ directions, and about $8 \mathrm{~nm}$ in the $z$ direction at $100 \mathrm{~ms}$ sampling interval.

Bead motility assay on suspended filaments-We used dielectrophoresis to stretch and suspend actin filaments across a trench etched between two gold electrodes $25,40,41$. We used optical tweezers to bring myosin X coated polystyrene beads (Polysciences, Inc.) (myosin:bead ratio $=10^{4}: 1$ ) onto the suspended actin filaments in M5Buffer (with $50 \mu \mathrm{M}$ MgATP) and an oxygen scavenging system. The beads were able to move freely along and about the suspended filaments, and we tracked their 3D positions using defocused images ${ }^{25}$, 40,41 .

\section{Supplementary Material}

Refer to Web version on PubMed Central for supplementary material.

\section{Acknowledgments}

This work was supported by the National Science Foundation NSEC grant DMR-0425780 through the Nano/Bio Interface Center and NIH grant GM086352. We acknowledge helpful discussion and comments by John H. Lewis and John. F. Beausang (University of Pennsylvania). We thank Dr. Tatyana M. Svitkina and Dr. Changsong Yang (University of Pennsylvania) for the TEM (NIH grant RR 22482) characterization of fascin-bundled actin and Dr. Kazuaki Homma (University of Massachusetts Medical School) for the GFP-Myosin X HMM construct.

\section{References}

1. Berg JS, Derfler BH, Pennisi CM, Corey DP, Cheney RE. Myosin-X, a novel myosin with pleckstrin homology domains, associates with regions of dynamic actin. J. Cell. Sci 2000;113:3439-3451. [PubMed: 10984435]

2. Berg JS, Cheney RE. Myosin-X is an unconventional myosin that undergoes intrafilopodial motility. Nat. Cell. Biol 2002;4:246-250. [PubMed: 11854753]

3. Tokuo H, Ikebe M. Myosin X transports Mena/VASP to the tip of filopodia. Biochem. Biophys. Res. Commun 2004;319:214-220. [PubMed: 15158464]

4. Zhang H, et al. Myosin-X provides a motor-based link between integrins and the cytoskeleton. Nat. Cell. Biol 2004;6:523-531. [PubMed: 15156152]

5. Sousa AD, Cheney RE. Myosin-X: a molecular motor at the cell's fingertips. Trends Cell Biol 2005;15:533-539. [PubMed: 16140532]

6. Bohil AB, Robertson BW, Cheney RE. Myosin-X is a molecular motor that functions in filopodia formation. Proc. Natl. Acad. Sci. USA 2006;103:12411-12416. [PubMed: 16894163]

7. Nagy S, et al. A myosin motor that selects bundled actin for motility. Proc. Natl. Acad. Sci. USA 2008;105:9616-9620. [PubMed: 18599451]

8. Kovacs M, Wang F, Sellers JR. Mechanism of Action of Myosin X, a Membrane-associated Molecular Motor. J. Biol. Chem 2005;280:15071-15083. [PubMed: 15705568]

9. Zhu X-J, et al. Myosin X regulates netrin receptors and functions in axonal path-finding. Nat. Cell Biol 2007;9:184-192. [PubMed: 17237772]

10. Pi X, et al. Sequential roles for myosin-X in BMP6-dependent filopodial extension, migration, and activation of BMP receptors. J. Cell Biol 2007;179:1569-1582. [PubMed: 18158328]

11. Weber KL, Sokac AM, Berg JS, Cheney RE, Bement WM. A microtubule-binding myosin required for nuclear anchoring and spindle assembly. Nature 2004;431:325-329. [PubMed: 15372037]

12. Toyoshima F, Nishida E. Integrin-mediated adhesion orients the spindle parallel to the substratum in an EB1- and myosin X-dependent manner. EMBO J 2007;26:1487-1498. [PubMed: 17318179]

13. Woolner S, O'Brien LL, Wiese C, Bement WM. Myosin-10 and actin filaments are essential for mitotic spindle function. J. Cell Biol 2008;182:77-88. [PubMed: 18606852] 
14. Zhang Z, Menq C-H. Three-dimensional particle tracking with subnanometer resolution using offfocus images. Appl. Opt 2008;47:2361-2370. [PubMed: 18449301]

15. Tokuo H, Mabuchi K, Ikebe M. The motor activity of myosin-X promotes actin fiber convergence at the cell periphery to initiate filopodia formation. J. Cell Biol 2007;179:229-238. [PubMed: 17954606]

16. Knight PJ, et al. The Predicted Coiled-coil Domain of Myosin 10 Forms a Novel Elongated Domain That Lengthens the Head. J. Biol. Chem 2005;280:34702-34708. [PubMed: 16030012]

17. Park H, et al. Full-Length Myosin VI Dimerizes and Moves Processively along Actin Filaments upon Monomer Clustering. Mol. Cell 2006;21:331-336. [PubMed: 16455488]

18. Homma K, Ikebe M. Myosin X Is a High Duty Ratio Motor. J. Biol. Chem 2005;280:29381-29391. [PubMed: 15961399]

19. Homma K, Saito J, Ikebe R, Ikebe M. Motor Function and Regulation of Myosin X. J. Biol. Chem 2001;276:34348-34354. [PubMed: 11457842]

20. Sun Y, et al. Myosin VI Walks "Wiggly"on Actin with Large and Variable Tilting. Mol. Cell 2007;28:954-964. [PubMed: 18158894]

21. Forkey JN, Quinlan ME, Corrie JET, Goldman YE. Single-molecule structural dynamics by fluorescence polarization microscopy. Biophys. J 1999;76:A20-A20.

22. Rosenberg SA, Quinlan ME, Forkey JN, Goldman YE. Rotational motions of macromolecules by single-molecule fluorescence microscopy. Acc. Chem. Res 2005;38:583-593. [PubMed: 16028893]

23. Forkey JN, Quinlan ME, Shaw MA, Corrie JET, Goldman YE. Three-dimensional structural dynamics of myosin V by single-molecule fluorescence polarization. Nature 2003;422:399-404. [PubMed: 12660775]

24. Yildiz A, et al. Myosin V Walks Hand-Over-Hand: Single Fluorophore Imaging with 1.5-nm Localization. Science 2003;300:2061-2065. [PubMed: 12791999]

25. Sun Y, McKenna JD, Murray JM, Ostap EM, Goldman YE. Parallax: High Accuracy ThreeDimensional Single Molecule Tracking Using Split Images. Nano Lett 2009;9:2676-2682. [PubMed: 19496608]

26. Rock RS, et al. A Flexible Domain Is Essential for the Large Step Size and Processivity of Myosin VI. Mol. Cell 2005;17:603-609. [PubMed: 15721263]

27. Yu C, et al. Myosin VI Undergoes Cargo-Mediated Dimerization. Cell 2009;138:537-548. [PubMed: 19665975]

28. Phichitha D, et al. Cargo binding induces dimerization of myosin VI. Proc Natl Acad Sci USA 2009;106:17320-17324. [PubMed: 19805065]

29. Iwaki M, et al. Cargo-Binding Makes a Wild-Type Single-Headed Myosin-VI Move Processively. Biophysical Journal 2006;90:3643-3652. [PubMed: 16500969]

30. Beausang, JF.; Sun, Y.; Quinlan, ME.; Forkey, JN.; Goldman, YE. Single Molecule Fluorescence Polarization via Polarized Total Internal Reflection Fluorescent Microscopy. In: Sialiano, I., editor. Laboratory Manual for Single Molecule Studies. Cold Spring Harbor, N.Y.: Cold Spring Harbor Laboratory; 2007.

31. Kerssemakers JW, et al. Assembly dynamics of microtubules at molecular resolution. Nature 2006;442:709-712. [PubMed: 16799566]

32. Ökten Z, Churchman LS, Rock RS, Spudich JA. Myosin VI walks hand-overhand along actin. Nat Struct \& Mol Biol 2004;11:884-887. [PubMed: 15286724]

33. Yildiz A, et al. Myosin VI Steps via a Hand-over-Hand Mechanism with Its Lever Arm Undergoing Fluctuations when Attached to Actin. J. Biol. Chem 2004;379:37222-37226.

34. Ishikawa R, Sakamoto T, Ando T, Higashi-Fujime S, Kohama K. Polarized actin bundles formed by human fascin-1: their sliding and disassembly on myosin II and myosin $\mathrm{V}$ in vitro. J Neurochem 2003;87:676-685. [PubMed: 14535950]

35. Vilfan A. Elastic Lever-Arm Model for Myosin V. Biophys. J 2005;88:3792-3805. [PubMed: 15792977]

36. Lan G, Sun SX. Flexible Light-Chain and Helical Structure of F-Actin Explain the Movement and Step Size of Myosin-VI. Biophys. J 2006;91:4002-4013. [PubMed: 16963511] 
37. Beausang JF, Schroeder HWI, Nelson PC, Goldman YE. Twirling of Actin by Myosins II and V Observed via Polarized TIRF in a Modified Gliding Assay. Biophys. J 2008;95:5820-5831. [PubMed: 18931255]

38. Sako Y, Minoguchi S, Yanagida T. Single-molecule imaging of EGFR signalling on the surface of living cells. Nat. Cell. Biol 2000;2:168-172. [PubMed: 10707088]

39. Ali MY, et al. Myosin V is a left-handed spiral motor on the right-handed actin helix. Nat. Struct. Biol 2002;9:464-467. [PubMed: 12006986]

40. Arsenault ME, Sun Y, Bau HH, Goldman YE. Using Electrical and Optical Tweezers to Facilitate Studies of Molecular Motors. Phys. Chem. Chem. Phys 2009;11:4834-4839. [PubMed: 19506758]

41. Arsenault ME, Zhao H, Purohit PK, Goldman YE, Bau HH. Confinement and Manipulation of Actin Filaments by Electric Fields. Biophys. J 2007;93:L42-L44. [PubMed: 17693465]

42. Mukherjea M, et al. Myosin VI Dimerization Triggers an Unfolding of a Three-Helix Bundle in Order to Extend Its Reach. Mol. Cell 2009:35.

43. Rock RS, et al. Myosin VI is a processive motor with a large step size. Proc. Natl. Acad. Sci. USA 2001;98:13655-13659. [PubMed: 11707568]

44. Choe S, Sun SX. The elasticity of $\alpha$-helices. J. Chem. Phys 2005;122:244912. [PubMed: 16035821]

45. Li, X-d; Jung, HS.; Mabuchi, K.; Craig, R.; Ikebe, M. The Globular Tail Domain of Myosin Va Functions as an Inhibitor of the Myosin Va Motor. J. Bio. Chem 2006;281:21789-21798. [PubMed: 16757473]

46. Thirumurugan K, Sakamoto T III, J.A.H. Sellers JR, Knight PJ. The cargo-binding domain regulates structure and activity of myosin 5. Nature 2006;442:212-215. [PubMed: 16838021]

47. Corrie JET, Craik JS, Munasinghe VRN. A homobifunctional rhodamine for labeling proteins with defined orientations of a fluorophore. Bioconjug. Chem 1998;9:160-167. [PubMed: 9548530]

48. Dunn AR, Spudich JA. Dynamics of the unbound head during myosin V processive translocation. Nat. Struct. Mol. Biol 2007;14:246-248. [PubMed: 17293871]

49. Pardee JD, Spudich JA. Purification of muscle actin. Methods. Cell. Biol 1982;24:271-289. [PubMed: 7098993]

50. Homma K, Saito J, Ikebe R, Ikebe M. $\mathrm{Ca}^{2+}$-dependent Regulation of the Motor Activity of Myosin V. J. Biol. Chem 2000;275:34766-34771. [PubMed: 10945977]

51. Harada Y, Sakurada K, Aoki T, Thomas DD, Yanagida T. Mechanochemical coupling in actomyosin energy transduction studied by in vitro movement assay. J. Mol. Biol 1990;216:49-68. [PubMed: 2146398]

52. Michel B, et al. Printing meets lithography: Soft approaches to high-resolution printing. IBM J. Res. Dev 2001;45:697-719.

53. Schmid H, Michel B. Siloxane Polymers for High-Resolution, High-Accuracy Soft Lithography. Macromol 2000;33:3042-3049.

54. Nitzsche B, Ruhnow F, Diez S. Quantum-dot-assisted characterization of microtubule rotations during cargo transport. Nat. Nanotech 2008;3:552-556. 

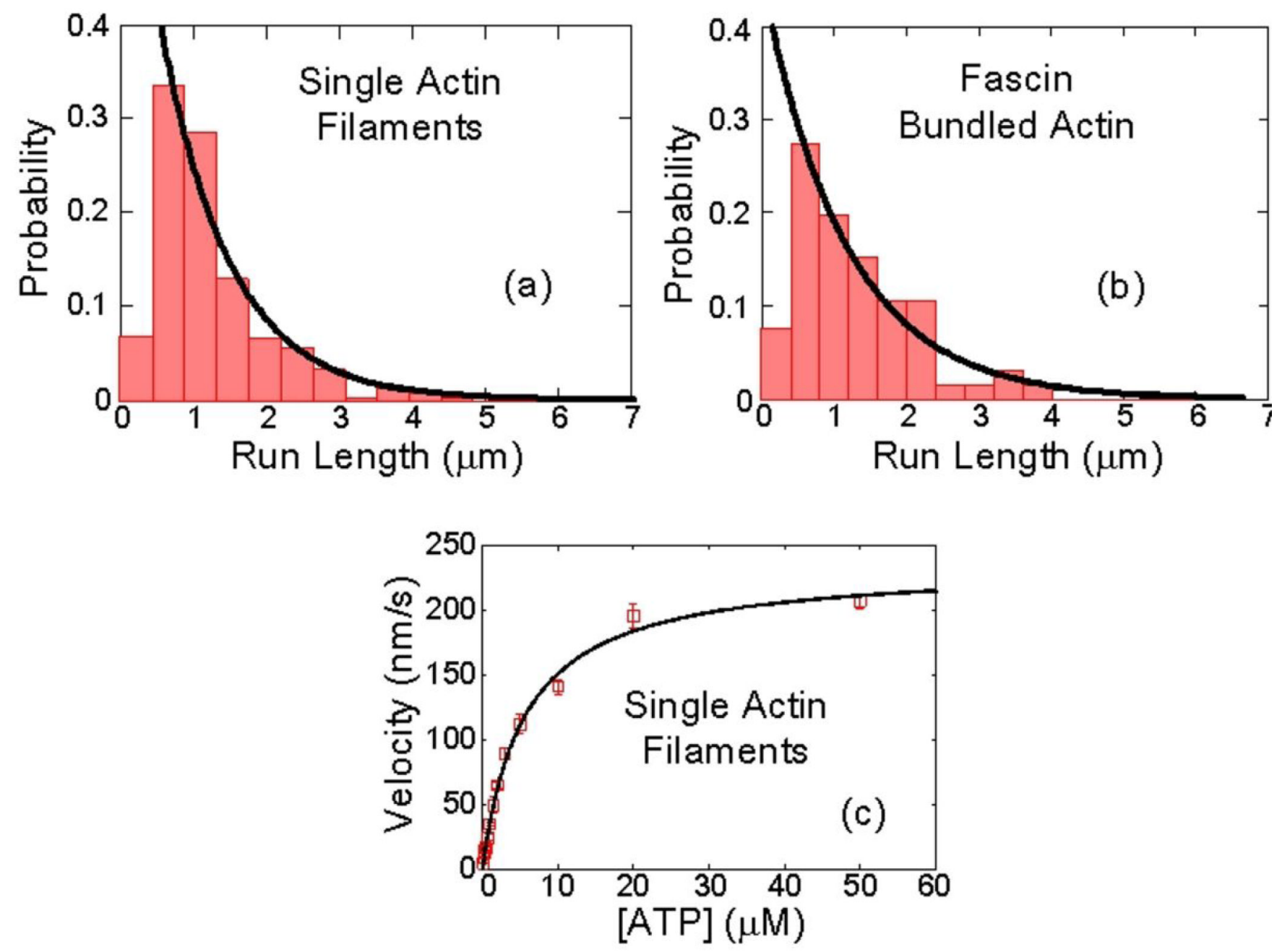

Figure 1.

Processivity and stepping rates of MyoX-LA-QD655 labeled myosin X on actin filaments and actin bundles. (a) The run-length distribution on suspended actin filaments. A single exponential fit (the first bin is excluded from the fitting) gives an average run length of 0.95 $\mu \mathrm{m}(95 \%$ confidence limits $+0.25 /-0.16 \mu \mathrm{m})$; (b) The run-length distribution on surface-bound actin bundles. A single exponential fitting (first bin excluded) gives an average run length of $1.16 \mu \mathrm{m}(95 \%$ confidence limits $+0.28 /-0.16 \mu \mathrm{m})$; (c) MgATP-dependence of the velocity of single MyoX-LA-QD655 on single immobilized actin filaments. Error bars are s.e.m. of the velocity. 

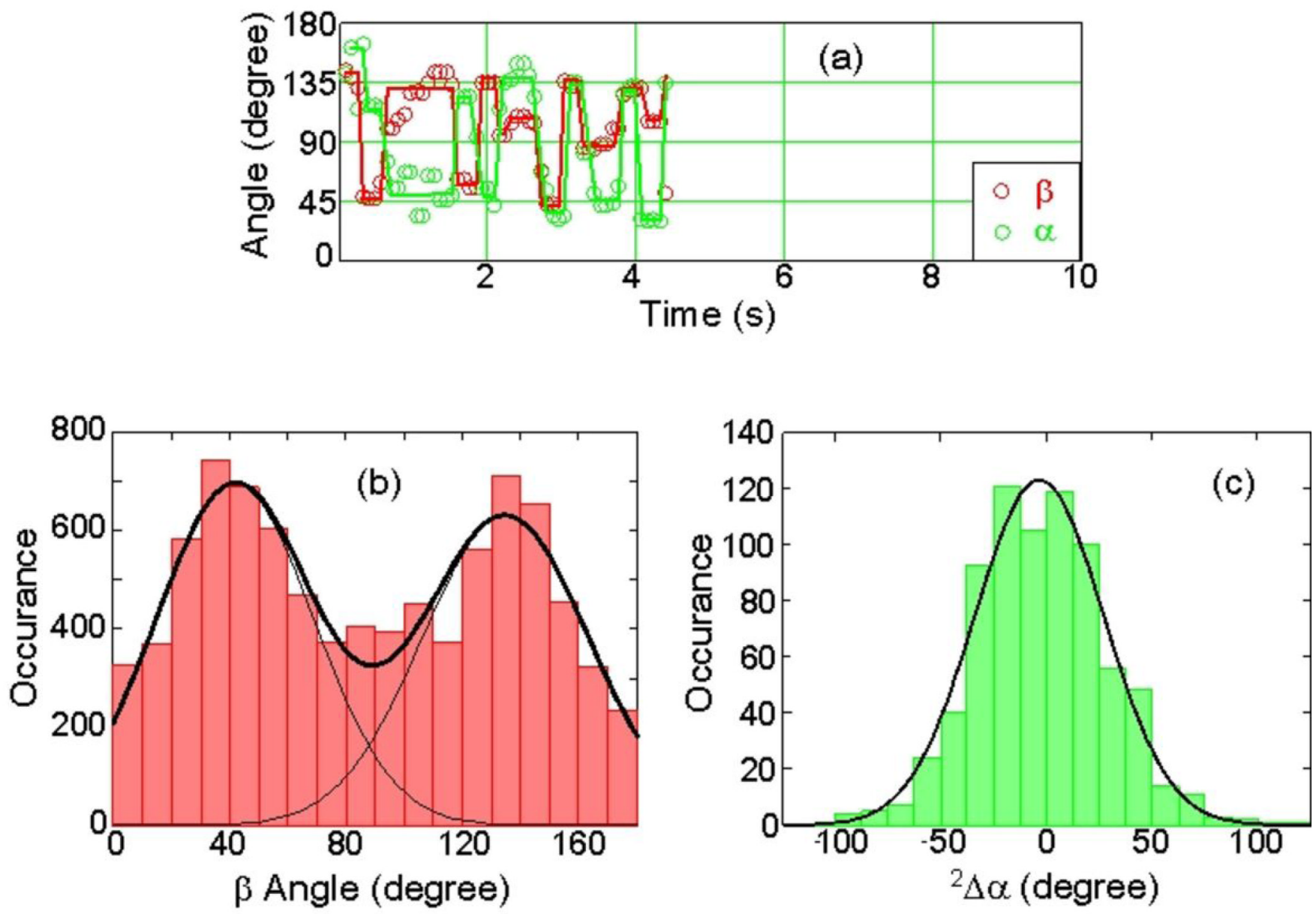

Figure 2.

polTIRF results for MyoX-BRCaM stepping on an actin filament immobilized on the glass surface. (a) A typical time course of $\beta$ (red, the axial angle of the BR probe relative to actin) and $\alpha$ (blue, the azimuthal angle of the probe around actin) transitions during myosin X motility at $2 \mu \mathrm{M} \mathrm{MgATP}$. Solid traces are Stepfinder fits ${ }^{31}$ to the angle recordings; (b) The distribution of $\beta$ angles. The two Gaussian fits $\left(42 \pm 27^{\circ}\right.$ (s.d., $n=4755$ ) and $135 \pm 28^{\circ}$ (s.d., $n=3948$ )) suggest that the myosin $\mathrm{X}$ lever arm switches between leading and trailing states; (c) Distribution of the changes of $\alpha$ after two consecutive steps (same-state angular change) ${ }^{2} \Delta \alpha$ $\left(-3.4 \pm 30^{\circ}\right.$ (s.d., $\left.n=757\right)$ ). 

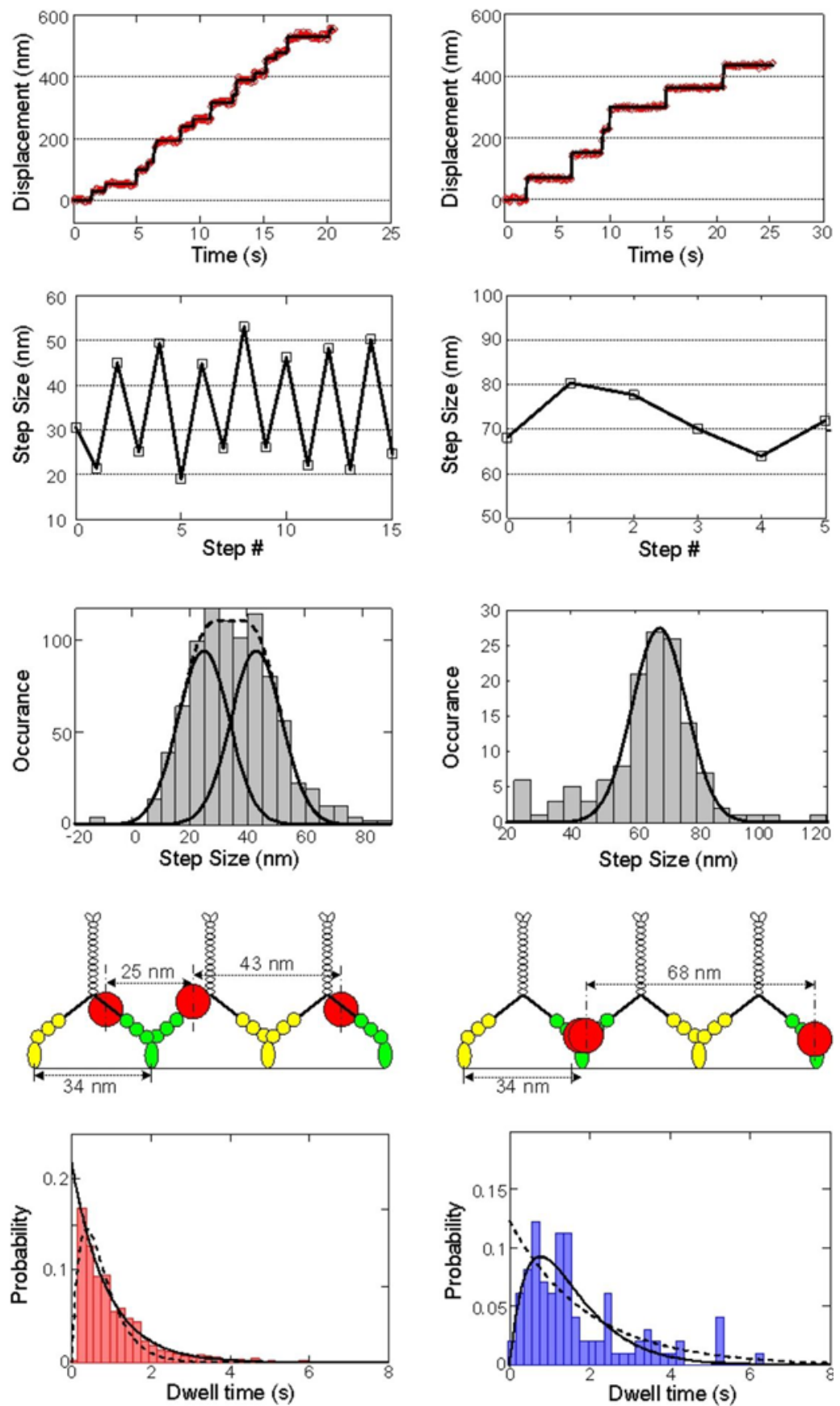

Figure 3.

Alternating step size supports the hand-over-hand model of myosin X. (a) About $86 \%$ of myosin X molecules showed alternating larger and smaller displacements. The step sizes in the second panel are measured from the molecule of first panel. The third panel shows the step size histogram of 66 alternating molecules. It is fitted with two Gaussian components of equal amplitude and width (solid curves, $25 \pm 9.4 \mathrm{~nm}$ and $43 \pm 9.4 \mathrm{~nm}$ (s.d.); the dashed curve is the sum of the two solid curves). Alternating large and small steps are expected from the cartoon in the fourth panel, where the quantum dot is drawn on the third CaM. The dwell time distribution, red bars, is fitted by a single exponential $P(t)=k \mathrm{e}^{-k t}$, yielding an average waiting time $(1 / k)$ of $0.89 \mathrm{~s}$ at $1 \mu \mathrm{M} \mathrm{MgATP}$. The dashed curve is a double exponential that doesn't 
approximate the data as well (F-test). (b) The rest of the myosin X molecules (14\%) showed larger step sizes of $68 \pm 8.4 \mathrm{~nm}$ (s.d., $n=142$ ). This behavior is expected from the cartoon in the fourth panel, where the quantum dot is drawn on the first $\mathrm{CaM}$. The dwell time distribution for the $0-68 \mathrm{~nm}$ case, blue bars, is fitted by a convolution of two single exponentials, $P(t)$ $=t k^{2} \mathrm{e}^{-k t}$, yielding an average waiting time $(2 / k)$ of $1.62 \mathrm{~s}$ at $1 \mu \mathrm{M} \mathrm{MgATP}$, about double the waiting time for the $25-43$ case. The dashed curve is a poorer fit by a single exponential decay (F-test). 

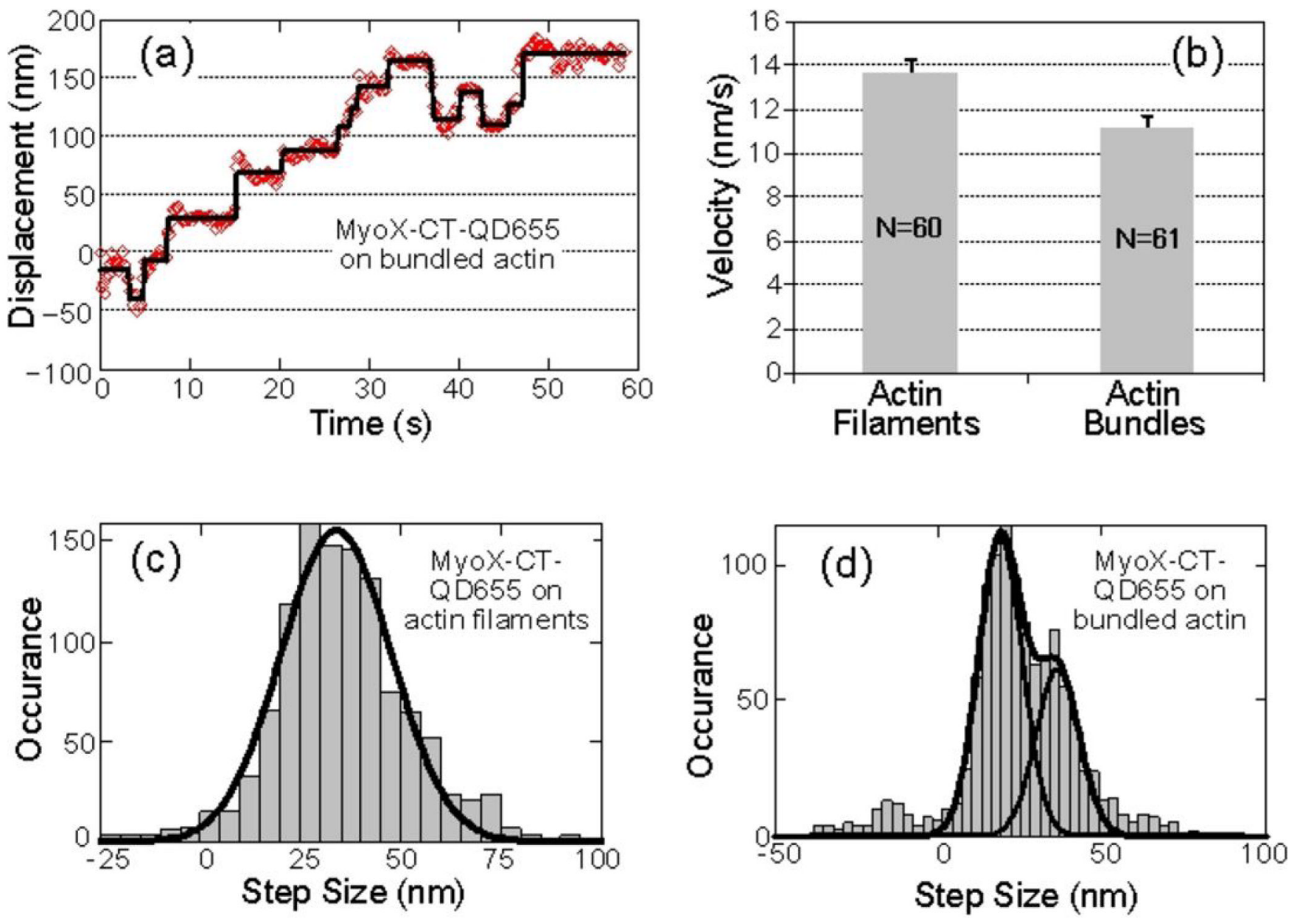

Figure 4.

MyoX-CT-QD655 exhibits different stepping behavior along actin filaments and bundles; (a) A stepping trace of myosin $\mathrm{X}$ on a single actin filament; (b) A stepping trace of myosin $\mathrm{X}$ on an actin bundle; (c) The step size distribution of MyoX-CT-QD655 on actin filaments with mostly forward steps (34 $\pm 14 \mathrm{~nm}$, s.d., $n=1105)$ and $4.5 \%$ backward steps $(-29 \pm 14$ nm, s.d., $n=53$ ); (d) The step size distribution of MyoX-CT-QD655 on actin bundles with mostly forward steps $(28 \pm 13 \mathrm{~nm}$, s.d., $n=1349)$ and $8 \%$ backward steps $(-17 \pm 7 \mathrm{~nm}, \mathrm{~s} . \mathrm{d} ., n=113)$, black curves. The data of forward steps were also fitted with two Gaussian components (magenta and green curves) with the center of magenta curve fixed at $34 \mathrm{~nm}$ for comparison with (c). $[\mathrm{MgATP}]=2 \mu \mathrm{M}$. 

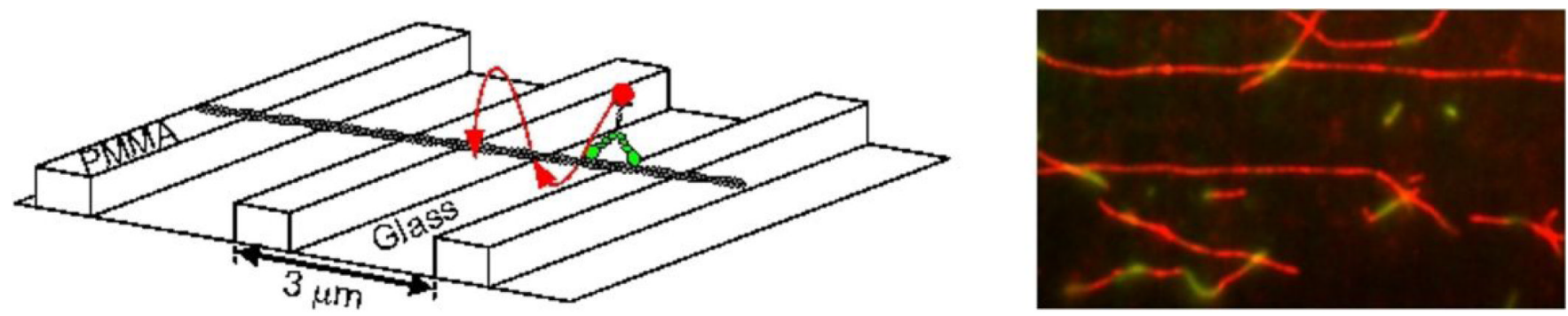

Figure 5.

Parallax tracking of myosin X molecules stepping along suspended actin filaments and bundles at $1 \mu \mathrm{M}$ MgATP. (a) myoX-LA-QD655 and (b) myoX-CT-QD655 step along an individual suspended actin filament; (c) myoX-CT-QD655 steps along a suspended actin bundle. Upper panels: displacement of myosin X along actins vs. time; Middle panels: displacements of QD655 on myosin X relative to actin in the $y(\Delta y$, blue $)$ and $z$ ( $\Delta z$, red) directions; Lower panels: 3-dimensional plots of the paths; (d) Path of a myosin X coated polystyrene bead stepping along a suspended actin filament at $50 \mu \mathrm{M}$ MgATP. In each case the path is a left-handed helix. 

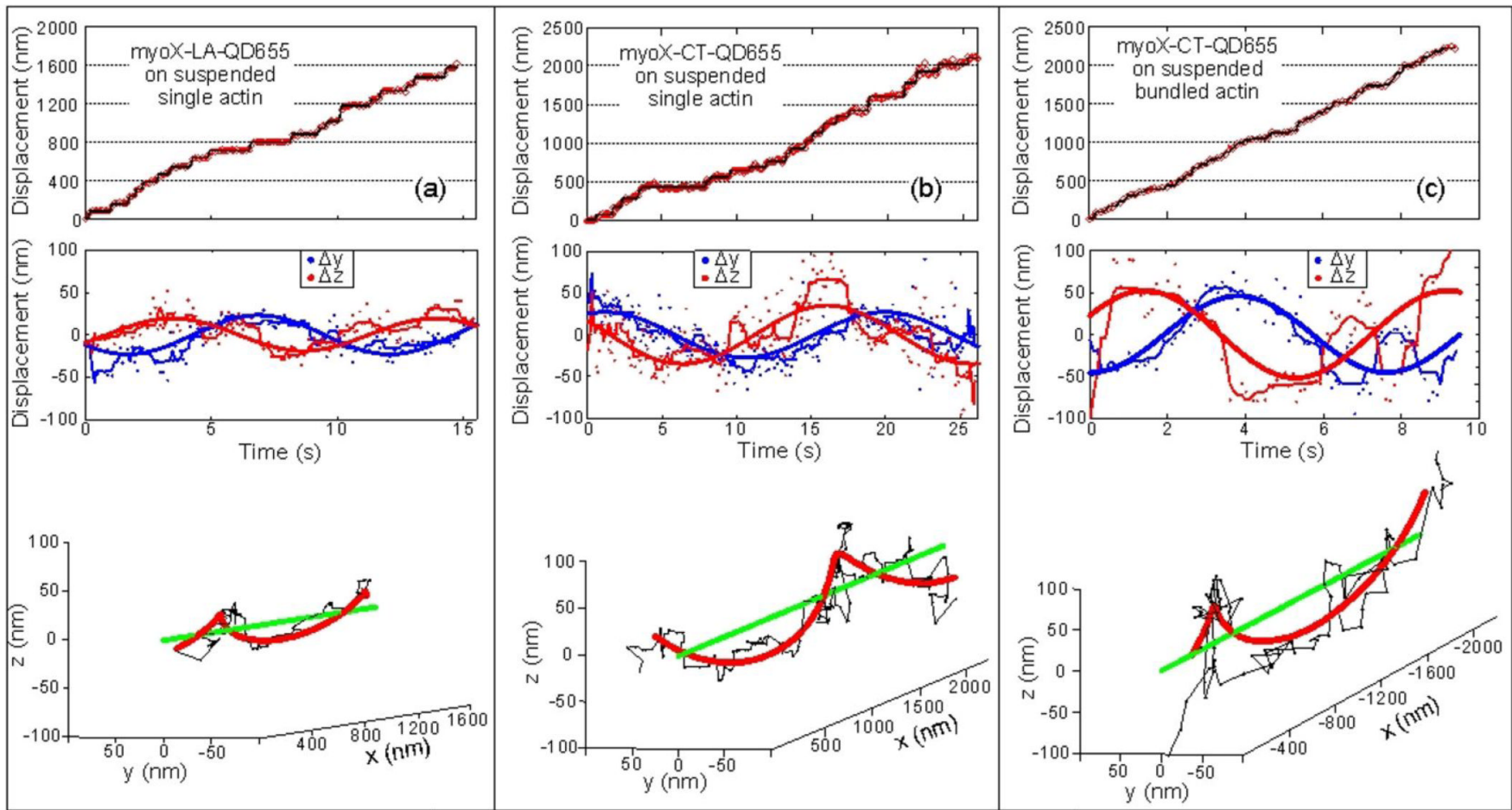

(d)

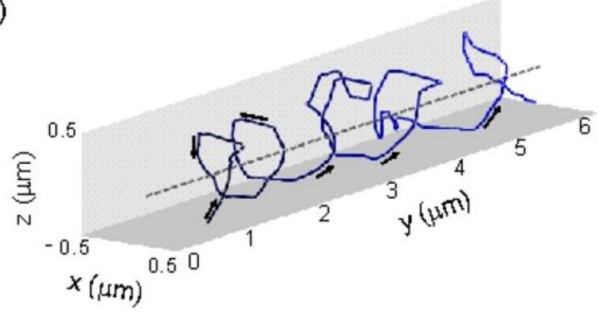

Figure 6.

Radial amplitudes of helical paths of myosin X molecules stepping along suspended actins. The cartoons are drawn approximately to scale to show the expected amplitudes of the QD655 helical paths in the three cases. The measured amplitude increases in the examples from left to right, but not as much as would be expected if the motor tail is straight and oriented radially. Error bars are s.e.m. of the radii. 


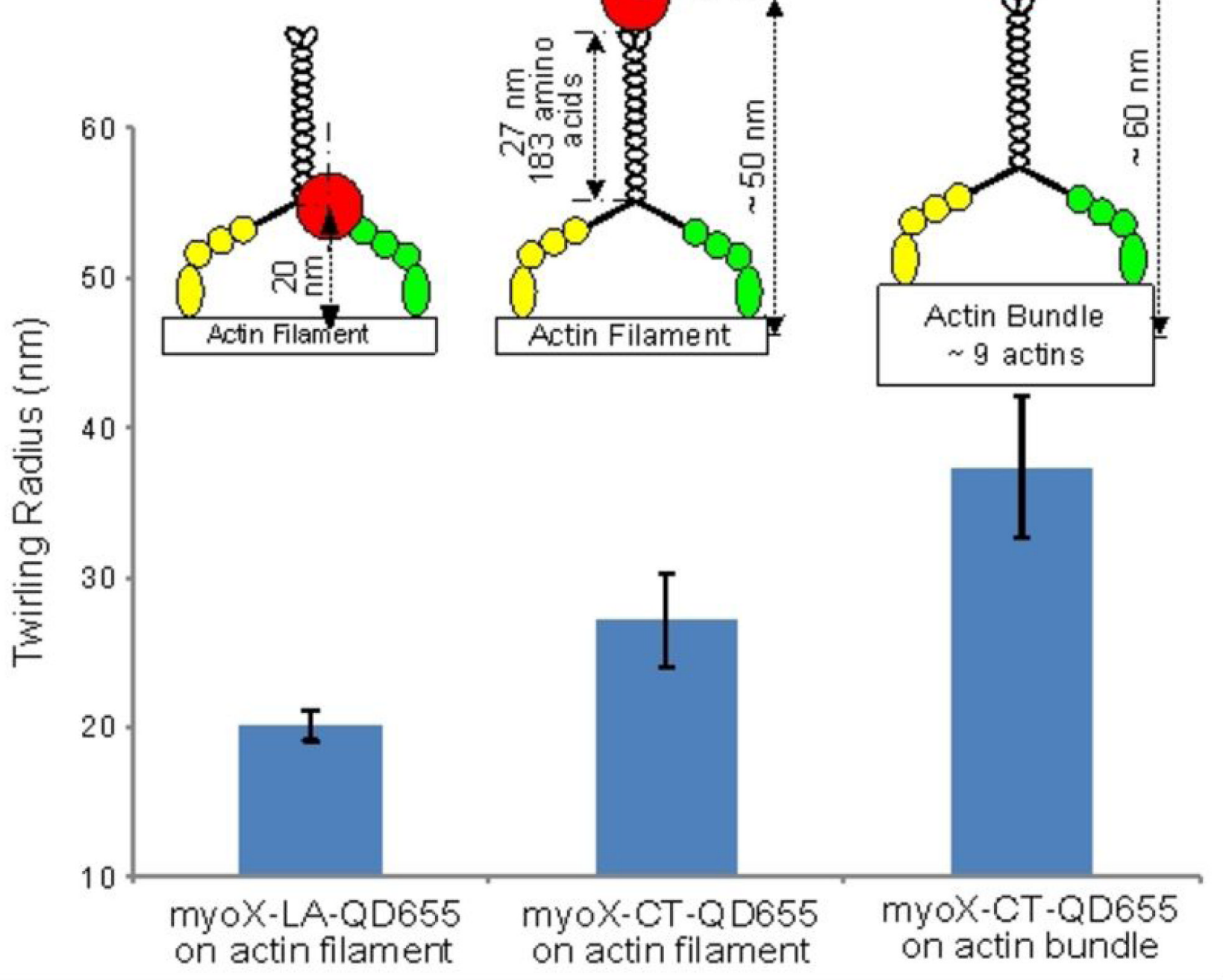

Figure 7. 